\title{
COMPORTAMENTO ESPACIAL DAS VARIÁVEIS PRODUÇÃO, VOLUME DE COPA E DIÂMETRO DE CAULE DA VARIEDADE MAXI GALA COM A UTILIZAÇÃO DA TÉCNICA DE COKRIGAGEM SOBRE POMAR COMERCIAL EM VACARIA-RS ${ }^{1}$
}

\author{
MÁRCIO EDUARDO BOEIRA BUENO², JARDEL TALAMINI DE ABREU ${ }^{3}$, \\ GERALDINE DE ANDRADE MEYER ${ }^{4}$, LEO RUFATO $^{5}$, LEONARDO JOSOÉ BIFFI $^{6}$
}

RESUMO - A Agricultura de Precisão (AP) permite a utilização de diferentes ferramentas na obtenção de informações, e estas otimizam a tomada de decisão por parte do produtor, impactando positivamente na receita final. O objetivo do trabalho foi verificar o comportamento das variáveis produção (PROD), volume de copa (VC) e diâmetro de caule (DC) da variedade Maxi Gala com a utilização da técnica da cokrigagem. $\mathrm{O}$ experimento foi conduzido em uma área de 0,90 hectare de produção comercial da variedade Maxi Gala, na Fazenda São Paulino, da empresa RASIP, em Vacaria-RS, apresentando como coordenadas geográficas $28^{\circ} 31^{\prime} 17^{\prime \prime}$ de latitude sul e 5049'17" de longitude oeste, durante as safras de 2010/2011 e 2011/2012. Coletaram-se 75 amostras para cada variável, em uma malha de $12 \mathrm{~m}$ na entrelinha e $10 \mathrm{~m}$ na linha. As variáveis avaliadas foram produção por planta, volume de copa e diâmetro de caule. Foram feitas a análise estatística descritiva dos dados e a análise espacial através dos semivariogramas. De posse dos modelos ajustados, realizou-se a interpolação pelo método da krigagem. Após, foi realizada a correlação simples dos parâmetros e elaborado os semivariogramas cruzados para interpolação, pela técnica da cokrigagem. Os parâmetros PROD versus DC apresentaram média correlação na variedade Maxi Gala, na safra de 2011. Nas safras de 2011 e 2012, os parâmetros VC versus DC também apresentaram média correlação. A técnica da cokrigagem pode ser uma ferramenta da AP a ser utilizada pela cultura da macieira no levantamento de informações. Verificou-se que houve resposta e redução na coleta de amostras das variáveis mais difíceis; na safra de 2011, reduziu-se a coleta de 15 amostras da PROD, e na safra de 2012, reduziu-se a coleta de 20 amostras do VC.

Termos para indexação: Malus domestica BORKH, Agricultura de Precisão, Geoestatistica, Krigagem, Semivariograma cruzado.

\section{SPACIAL BEHAVIOR OF VARIABLES: PRODUCTION, CANOPY VOLUME AND DIAMENTER RANGE OF MAXI GALA WITH THE USE OF COKRIGING TECHNIQUE ON COMERCIAL ORCHARD IN VACARIA-RS}

\begin{abstract}
Precision Agriculture (PA) allows the use of different tools in obtaining information, and they optimize decision making by producers having a positive impact on the final recipe. The objective was to verify the behavior of the production variables (PROD), canopy (CV) and stem diameter (SD) variety Maxi Gala with the use of the cokriging technique. The experiment was conducted in an area of 0.90 hectares of commercial production of the variety Maxi Gala at Fazenda São Paulino RASIP Company in Vacaria - RS presenting as geographical coordinates $28^{\circ} 31^{\prime} 17^{\prime \prime}$ ' south latitude and $50^{\circ} 49^{\prime} 17^{\prime \prime}$ ' west longitude during 2010/11 and 2011/12 crops. It was collected 75 samples for each variable in a loop of $12 \mathrm{~m}$ between line and $10 \mathrm{~m}$ on the line. The variables evaluated were yield per plant, canopy volume and diameter. Descriptive statistical analysis and spatial analysis through the semivariogram were made. By having the adjusted models, it was held the interpolation by the kriging method. After it was performed a simple correlation of the parameters, prepared the crossed semivariogram for the interpolation by the cokriging technique. The PROD versus SD parameters showed an average correlation in the Maxi Gala cultivar in 2011 harvest. In 2011 and 2012 harvests the $\mathrm{CV}$ versus SD parameters also showed an average correlation. The cokriging technique can be a PA tool to be used by apple culture in gathering information. It was found that there was response and reduction in collecting samples of the most difficult variables, in the 2011 harvest 15 samples of PROD were reduced and in the 2012 harvest 20 samples of the $\mathrm{CV}$ were reduced.

Index terms: Malus domestica Borkh, Precision Agriculture, Geostatistics, Kriging, cross semivariogram.

${ }^{1}$ (Trabalho 128-14). Recebido em: 01-04-2014. Aceito para publicação em: 30-03-2015.

${ }^{2} \mathrm{Eng}^{\mathrm{o}} \mathrm{Agr}^{\mathrm{o}}$ M.Sc do Programa de Pós Graduação em Produção Vegetal / UDESC - CAV. E-mail:marciobueno2003@yahoo.com.br

${ }^{3}$ Tecnol. Frut. Mestrando do Programa de Pós Graduação em Produção Vegetal / UDESC - CAV. E-mail: jtabreu@gmail.com

${ }^{4} \mathrm{Eng}^{\mathrm{a}} \mathrm{Agr}^{\mathrm{a}} \mathrm{Dr}^{\mathrm{a}}$. do Programa de Pós Graduação em Produção Vegetal / UDESC - CAV. E-mail: ge_meyer@hotmail.com

${ }^{5}$ Eng $^{\mathrm{o}}$ Agr $^{\mathrm{o}}$ Dr. em Fruticultura, CAV - UDESC. E-mail: leoruffato@yahoo.com.br

${ }^{6} \mathrm{Eng}^{\mathrm{o}} \mathrm{Agr}^{\mathrm{o}}$ M.Sc. Produção Vegetal, CAV - UDESC. E-mail; leonardo_biffi@yahoo.com.br
\end{abstract}




\section{INTRODUÇÃO}

O agronegócio é um setor que contribui de forma significativa ao PIB brasileiro, abrindo novas divisas e gerando renda e emprego. Neste contexto, a cultura da macieira merece destaque por ocupar uma posição importante entre os grandes produtores mundiais.

Além da tradição de mais de 30 anos no cultivo comercial da fruta, fatores como a produção de variedades modernas, disponibilidade de terras, regiões com condições climáticas favoráveis, bem como preocupações com produtividade, infraestrutura de embalagem e conservação, transformaram o Brasil em um grande produtor mundial (BITTENCOURT et al., 2011).

Fioravanço (2009) destaca que a tecnologia foi um fator importante e decisivo para a competitividade da maçã brasileira no mercado externo, sendo que o aumento da produtividade e a melhoria da qualidade do produto estão fortemente relacionados com inovações tecnológicas e com sua adoção.

Porém, com o passar dos anos, o custo de produção aumentou, e o preço pago por quilo de maçã ao produtor não aumentou na mesma proporção. Este fato fez com que muitos produtores abandonassem a atividade, e o setor vivenciasse uma crise. A partir deste episódio, modificou-se a gestão dos pomares, buscando aumentar a eficiência do processo de produção.

Tentando reduzir os custos de produção, hoje a cultura da macieira busca um perfil mecanizado nos diferentes manejos do pomar, devido principalmente à necessidade de uso da mão de obra que impacta, em média, 60\% dos custos de produção. Esta mecanização já existe para alguns manejos do pomar e está sendo pesquisada e testada por órgãos oficiais. Um exemplo são as plataformas de colheita, raleio e poda.

Nesta perspectiva, a agricultura de precisão (AP) e suas diferentes ferramentas podem ser úteis no sentido de geração de informações para manejos mais precisos e eficientes, e diminuição do uso de mão de obra no levantamento dessas informações.

Entre as ferramentas da AP, a técnica da cokrigagem, segundo Landin et al. (2011), pode ser utilizada em situações em que a determinação de variáveis é cara e de difícil amostragem, comprometendo assim o estudo da variabilidade espacial de tal variável. Para esses casos, aplica-se a técnica da cokrigagem, que tem por objetivo descrever a variabilidade espacial e/ou temporal simultânea entre duas variáveis aleatórias, sendo que uma delas, conhecida como secundária, deve ser de fácil amostragem e/ou baixo custo de obtenção e apresentar alta correlação espacial com a variável de difícil determinação, conhecida como variável primária, e sobre a qual se deseja estimar valores.

O conhecimento sobre a técnica da cokrigagem no levantamento de parâmetros fitotécnicos, na cultura da macieira, é ainda insuficiente. Desta forma, este trabalho teve por objetivo verificar o comportamento das variáveis produção (PROD), volume de copa (VC) e diâmetro de caule (DC) da variedade Maxi Gala com a utilização da técnica da cokrigagem.

\section{MATERIAS E MÉTODOS}

O experimento foi conduzido em uma área de produção comercial da variedade Maxi Gala, da Fazenda São Paulino, da empresa Randon Agrossilvopastoril S.A. (RASIP), em Vacaria $\mathrm{RS}$, apresentando como coordenadas geográficas 28 31'17' de latitude sul e 5049'17' de longitude oeste, durante as safras de 2010/2011 e 2011/2012.

A área analisada é ocupada com $66 \%$ da variedade Maxi Gala e 34\% com a variedade Fuji Moore, plantadas no ano de 2006, dispostas em quatro fileiras de Maxi Gala, para duas fileiras de Fuji Moore. As variedades foram enxertadas sobre porta-enxerto marubakaido com filtro de EM9, tendo em média 3,5 $\mathrm{m}$ de altura. $\mathrm{O}$ espaçamento para a variedade Maxi Gala é de $4 \mathrm{~m}$ entre linhas e $1 \mathrm{~m}$ entre plantas, e para a variedade Fuji Moore, é de 4 $\mathrm{m}$ entre linhas e 1,20 m entre plantas.

Amostrou-se de forma regular na linha de plantas, formando uma malha de $12 \mathrm{~m}$ na entrelinha e $10 \mathrm{~m}$ na linha. O número de plantas amostradas foi de 75 plantas, representando uma área de 0,90 hectare.

Utilizou-se, para o georreferenciamento das plantas amostradas, um GNSS (Global Navigation Satellite Systems), marca Topcon (GPS Classe IV), modelo Hiper Lite+, com dupla frequência L1+L2, usando o sistema GPS (Americano) e o sistema GLONASS (Russo), com acurácia inferior a 0,5 m. Os dados foram levantados pelo aparelho no Datum SIRGAS 2000, projeção UTM, fuso 22, Hemisfério Sul.

A colheita dos frutos foi realizada no mês de fevereiro de 2011 e 2012, sendo a variável PROD determinada pela multiplicação de forma direta do número de frutos por planta e a massa média de frutos. O número de frutos por planta foi determinado através da contagem de forma visual, uma semana antes da colheita das maçãs. Esses dados foram anotados em uma planilha e depois digitados em uma planilha eletrônica. Na massa média de frutos, coletou-se, nos quatro quadrantes da planta, uma amostra de 30 frutos. Pesou-se a amostra (PA) e 
dividiu-se pelo número de frutos que ela formava (NF) para obter a massa média de frutos por planta. $\mathrm{Na}$ safra de 2012, não se conseguiu a informação PROD, pois a empresa havia colhido parte do experimento e não foi possível obter o número de frutos por planta.

As variáveis $\mathrm{VC}$ e DC foram determinadas no mês de julho de 2011 e 2012. No VC, as medições foram realizadas com o auxílio de uma régua de madeira com $4 \mathrm{~m}$ de comprimento. Mediu-se a altura da copa (m), a partir do ponto de inserção do primeiro ramo no tronco, largura $(\mathrm{m})$ e o comprimento da copa (m) e multiplicaram-se as três informações. O DC foi obtido através da média das medidas longitudinal e transversal à linha de plantio, do diâmetro do tronco a $20 \mathrm{~cm}$ do solo, aproximadamente, e acima do ponto de enxertia, com o auxílio de um paquímetro graduado em milímetros.

Os parâmetros foram avaliados através da estatística descritiva por meio do software WinStat versão 2.0. Determinou-se a normalidade dos parâmetros através do teste de KolmogorovSmirnov, a 5\% de probabilidade. As análises geoestatísticas foram realizadas no programa GS+ versão 7.0, ajustando-se o "lag" que apresentava o melhor coeficiente de determinação $\left(\mathrm{r}^{2}\right)$. $\mathrm{O}$ grau de dependência espacial das variáveis foi analisado com base na classificação de Cambardella et al. (1994), em que são considerados de forte dependência espacial os semivariogramas com efeito pepita de $25 \%$ do patamar, moderada quando entre $25 \%$ e $75 \%$ e de fraca quando $>75 \%$. A interpolação dos dados foi feita por krigagem ordinária, usando-se o software Surfer 8.02 - Surface Mapping System. As correlações foram determinadas com base no método de correlação de Pearson, ao nível de probabilidade de $5 \%$, por meio do software Winstat versão 2.0.

\section{RESULTADOS E DISCUSSÃO}

Os parâmetros foram analisados por meio da estatística descritiva (Tabela 1), e de acordo com o teste de Kolmogorov-Smirnov, a 5\% de probabilidade, a PROD apresentou normalidade em sua distribuição, na safra de 2011. O DC, nos dois anos, apresentou comportamento normal de distribuição, e o VC não apresentou normalidade em sua distribuição, nos dois anos.

A importância da verificação de distribuição normal ou não normal dos dados permite averiguar se existem valores extremos (outlier máximo e outlier mínimo), que destoam do conjunto de dados, possibilitando, dessa maneira, a exclusão de valores discrepantes que podem ser decorrentes de erros de amostragem ou de erros analíticos (SIQUEIRA et al., 2009).

Por meio da análise descritiva, observou-se que o $\mathrm{CV}$ obtido dos dados permitiu caracterizar, nas safras de 2011 e 2012, a PROD, o VC e o DC como média variação $(12 \leq \mathrm{CV} \leq 60 \%)$ (WARRICK; NIELSEN,1980).

Nas safras de 2011 e 2012, conforme Tabela 2, o modelo de semivariograma que melhor se ajustou, pelo coeficiente de Akaike, ao modelo experimental dos parâmetros avaliados, foi o modelo gaussiano.

Verifica-se que o alcance, na safra de 2011, nos parâmetros avaliados, variou de aproximadamente $11,20 \mathrm{~m}$ a $13,5 \mathrm{~m}$. Portanto, para garantir a dependência espacial, pontos de amostragem deveriam ser coletados a uma distância menor que o alcance. Já na safra de 2012, o alcance variou de aproximadamente $13,7 \mathrm{~m}$ a $15,4 \mathrm{~m}$. Com relação ao efeito pepita $(\mathrm{Co})$, pode-se analisar o grau de dependência espacial, utilizando a classificação proposta por Cambardella et al. (1994). Os parâmetros avaliados para as safras de 2011 e 2012 apresentaram forte dependência espacial.

Os resultados da análise de correlação entre os parâmetros avaliados, para as safras de de 2011 e 2012, estão representados na Tabela 3.

Com base nos coeficientes de Pearson proposto por Beiguelman (1996), observou-se, na safra de 2011, correlação média da PROD com o DC e VC com DC 0,59 e 0,57 , respectivamente.

Na safra de 2012, confirmou-se a correlação média entre VC com DC 0,57 , porém não houve a confirmação da PROD com o DC, porque a empresa colheu parte do experimento e faltou o levantamento do parâmetro número de frutos por planta utilizado no cálculo da produção.

Biffi (2008) evidenciou alta correlação entre as variáveis produção e diâmetro de caule em maçã, encontrando altas produções onde havia diâmetros de caule maiores que a média.

Para o semivariograma cruzado PROD versus DC, da safra de 2011 (Figura 1a), o modelo gaussiano foi o que mais se ajustou, apresentando alcance de $16,50 \mathrm{~m}$ com coeficiente de determinação de 0,80 . Nesta situação, houve dependência entre as amostras.

Pela cokrigagem, o DC utilizado como covariável provocou alteração no alcance da dependência espacial, assim como no patamar do semivariograma da PROD. O alcance aumentou $3 \mathrm{~m}$, e o patamar $(\mathrm{Co}+\mathrm{C})$ diminui $46 \%$. Os demais parâmetros mantiveram-se com pequena variação.

$\mathrm{Na}$ Tabela 4, foi apresentada a cokrigagem entre a variável principal PROD e a covariável DC. Foram retiradas 5; 10 e 15 amostras, sempre 
demonstrando dependência espacial entre os parâmetros. Quando se retiraram 20 amostras, não houve dependência espacial entre eles.

Para o semivariograma cruzado VC versus DC, da safra de 2011 (Figura 1B), o modelo que mais se ajustou foi o esférico, com alcance de 17,10 m e com coeficiente de determinação de 0,01 . Nesta situação, apesar das variáveis possuírem correlação, não houve dependência das amostras.

Para o semivariograma cruzado VC versus DC, da safra de 2012 (Figura 2), o modelo gaussiano foi o que mais se ajustou, apresentando alcance de $14,20 \mathrm{~m}$ com coeficiente de determinação de 0,71 .
Nesta situação, houve dependência entre as amostras. Pela cokrigagem, o DC utilizado como covariável provocou alteração no alcance da dependência espacial, assim como no patamar do semivariograma do VC. O alcance diminui 1,70 m, o patamar $(\mathrm{Co}+\mathrm{C})$ aumentou $72 \%$. A dependência espacial manteve-se forte, e o coeficiente de determinação reduziu $0,07 \%$.

$\mathrm{Na}$ Tabela 5, foi apresentada a cokrigagem entre VC e DC. Foram retiradas 5; 10; 15 e 20 amostras, sempre demonstrando dependência espacial entre os parâmetros. Quando se retiraram 25 amostras, não houve dependência espacial entre eles.

TABELA 1 - Estatística descritiva dos parâmetros produção, volume de copa e diâmetro de caule da variedade Maxi Gala, nas safras de 2011 e 2012. Vacaria, 2013.

\begin{tabular}{|c|c|c|c|c|c|c|}
\hline Variáveis & PROD & $\mathrm{VC}$ & DC & PROD & $\mathrm{VC}$ & DC \\
\hline & \multicolumn{3}{|c|}{ Safra 2011} & \multicolumn{3}{|c|}{ Safra 2012} \\
\hline $\mathrm{N}^{\circ}$ amostras & 75,00 & 75,00 & 75,00 & & 75,00 & 75,00 \\
\hline Média & 31,92 & 8,84 & 59,91 & & 9,61 & 67,46 \\
\hline Mediana & 31,64 & 8,87 & 59,00 & & 9,44 & 67,00 \\
\hline Mínimo & 11,34 & 4,58 & 39,00 & & 5,72 & 43,00 \\
\hline Máximo & 64,90 & 12,58 & 79,00 & & 12,58 & 87,50 \\
\hline Variância & 100,21 & 2,19 & 65,79 & & 2,28 & 88,06 \\
\hline DP & 10,01 & 1,48 & 8,11 & & 1,51 & 9,38 \\
\hline Assimetria & 0,49 & 0,19 & 0,48 & & $-0,23$ & 0,11 \\
\hline Curtose & 1,36 & 1,36 & 0,05 & & 0,12 & $-0,42$ \\
\hline CV $(\%)$ & 31,36 & 16,73 & 13,54 & & 15,71 & 13,91 \\
\hline Ks $0.05 \%$ & 0,15 & 0,05 & 0,15 & & 0,01 & 0,15 \\
\hline Ks & 0,08 & $0.1^{*}$ & 0,07 & & $0.12 *$ & 0,08 \\
\hline
\end{tabular}

PROD (produção), VC (volume de copa); DC(diâmetro de caule); DP (desvio padrão); CV(coef. de variação); As (coef. Assimetria), K(coef. Curtose); teste de Kolmogorov-Sirnov (KS).

TABELA 2 - Semivariogramas ajustados dos parâmetros: produção (PROD), volume de copa (VC) e diâmetro de caule (DC), da variedade Maxi Gala, nas safras de 2011 e 2012. Vacaria, 2013.

\begin{tabular}{ccccccccc}
\hline Variáveis & Modelo & $\mathrm{C}$ & $\mathrm{Co}$ & $\mathrm{Co}+\mathrm{C}$ & $\mathrm{Co} /(\mathrm{Co}+\mathrm{C})$ & $\mathrm{Ao}$ & Classif. & $\mathrm{r}^{2}$ \\
\hline & \multicolumn{8}{c}{$\begin{array}{c}\text { Safra } 2011 \\
\end{array}$} \\
PROD $(\mathrm{kg} / \mathrm{pl})$ & GAUS & 104,20 & 0,100 & 104,30 & 0,0009 & $\mathrm{~m}$ & & \\
VC $\left(\mathrm{m}^{3}\right)$ & GAUS & 1,92 & 0,336 & 2,26 & 0,1487 & 11,20 & Forte & 0,89 \\
DC $(\mathrm{mm})$ & GAUS & 65,89 & 0,100 & 65,99 & 0,0010 & 13,00 & Forte & 0,82
\end{tabular}

Safra 2012

\begin{tabular}{ccccccccc} 
PROD $(\mathrm{kg} / \mathrm{pl})$ & & & & & & & & \\
$\mathrm{VC}\left(\mathrm{m}^{3}\right)$ & GAUS & 1,59 & 0,001 & 1,59 & 0,0006 & 15,40 & Forte & 0,92 \\
$\mathrm{DC}(\mathrm{mm})$ & GAUS & 71,47 & 0,100 & 71,57 & 0,0014 & 13,70 & Forte & 0,70 \\
\hline
\end{tabular}

Efeito Pepita (Co): semivariância no ponto zero; Patamar $(\mathrm{Co}+\mathrm{C})$ : semivariância a um espaço maior que a distância do alcance; Alcance (Ao): distância após a qual os valores das propriedades não são espacialmente correlacionados; Modelos ajustados aos semivariogramas experimentais: Gaussiano ; Dependência espacial (forte $<25 \%$; moderada 25 a $75 \%$; fraca $>75 \%$ a 100 e $>100$ efeito pepita puro)

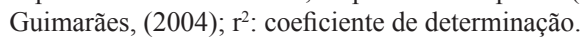


TABELA 3 - Coeficiente de correlação linear de Pearson (R) para parâmetros produção (PROD), volume de copa (VC) e diâmetro de caule (DC), da variedade Maxi Gala, nas safras de 2011 e 2012. Vacaria, 2013.

\begin{tabular}{llll}
\hline & PROD & VC & DC \\
\hline \hline \multicolumn{4}{c}{ Safra 2011} \\
PROD & 1,000 & & \\
VC & 0,153 & 1,000 & \\
DC & 0,595 & 0,568 & 1,000 \\
\hline \multicolumn{5}{c}{ Safra 2012} \\
PROD & 0,000 & \\
VC & 0,000 & 1,000 & \\
DC & 0,000 & 0,575 & 1,000 \\
\hline
\end{tabular}

PROD (produção por planta), VC (volume de copa) e DC(diâmetro de caule).

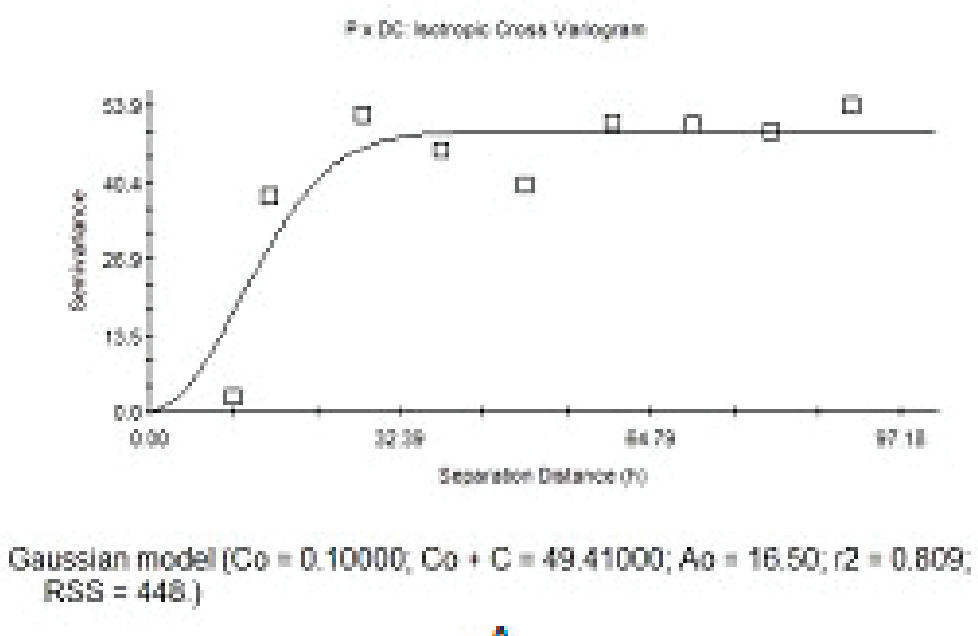

A

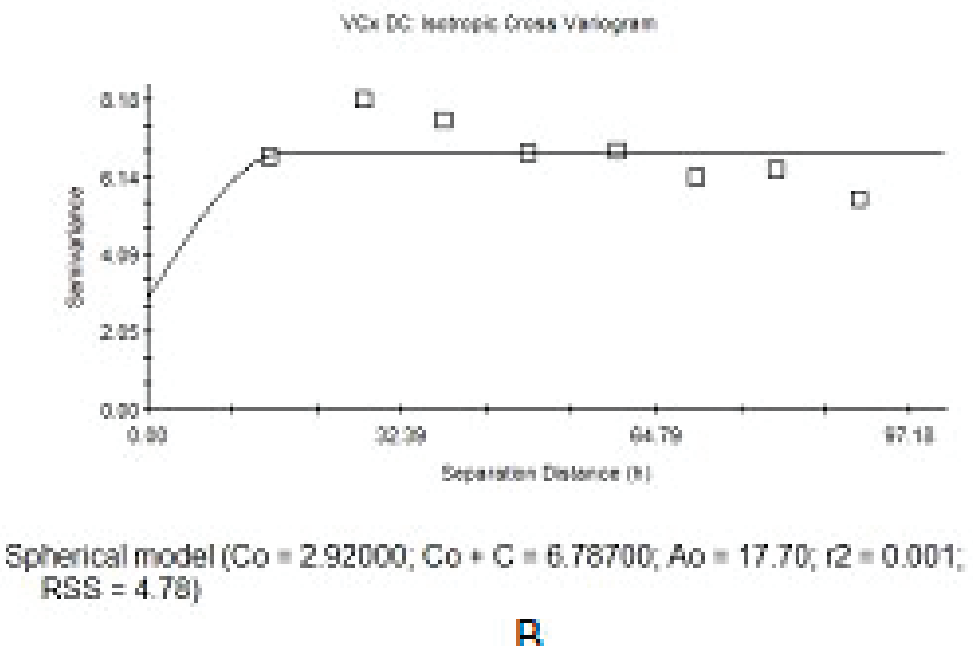

B

FIGURA 1 - Semivariogramas cruzados de produção versus diâmetro de caule (A) e volume de copa versus diâmetro de caule (B), safra de 2011. Vacaria, 2013. 
TABELA 4 - Parâmetros dos semivariogramas cruzados ajustados das variáveis: produção (PROD) versus diâmetro de caule (DC) com números diferentes de amostra de produção (PROD) da variedade Maxi Gala, na safra de 2011. Vacaria, 2013.

\begin{tabular}{ccccccccc}
\hline Variáveis & Modelo & C & Co & Co $+\mathrm{C}$ & $\mathrm{Co} /(\mathrm{Co}+\mathrm{C})$ & Ao & Classif. & $\mathrm{r}^{2}$ \\
\hline & \multicolumn{8}{c}{$\%$} \\
PROD & GAUS & 104,20 & 0,10 & 104,30 & 0,0009 & 13,50 & Forte & 0,89 \\
PRODxDC & GAUS & 49,31 & 0,10 & 49,41 & 0,002 & 16,50 & Forte & 0,80 \\
PRODxDC (-5) & GAUS & 53,38 & 0,10 & 53,48 & 0,001 & 16,60 & Forte & 0,80 \\
PRODxDC (-10) & GAUS & 53,19 & 0,10 & 53,29 & 0,001 & 16,50 & Forte & 0,80 \\
PRODxDC (-15) & GAUS & 55,94 & 0,10 & 56,04 & 0,001 & 16,50 & Forte & 0,76 \\
PRODxDC (-20) & - & 0,00 & 59,90 & 59,90 & 1 & 90,70 & E.P. Puro & 0,22 \\
\hline
\end{tabular}

Efeito Pepita (Co): semivariância no ponto zero; Patamar $(\mathrm{Co}+\mathrm{C})$ : semivariância a um espaço maior que a distância do alcance; $\mathrm{Al}-$ cance (Ao): distância após a qual os valores das propriedades não são espacialmente correlacionados; Dependência espacial (forte < $25 \%$; moderada 25 a $75 \%$; fraca $>75 \%$ a 100 e $>100$ efeito pepita puro). Guimarães (2004); $\mathrm{r}^{2}$ : coeficiente de determinação. Modelo ajustado aos semivariogramas experimentais: Gaussiano.

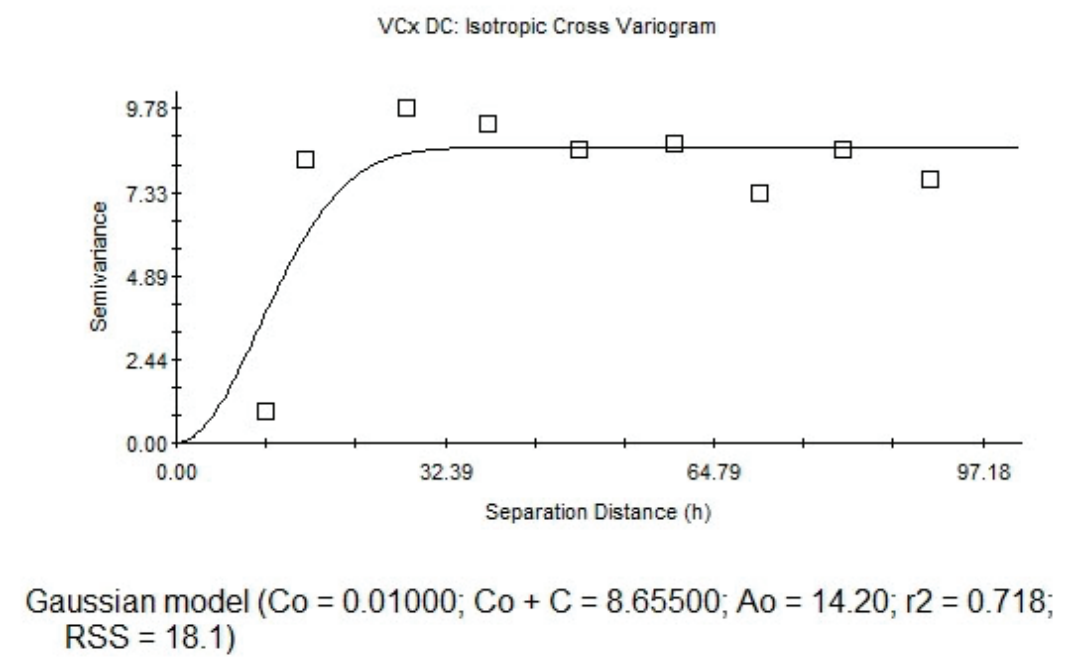

FIGURA 2- Semivariograma cruzado do volume de copa versus diâmetro de caule, safra de 2012. Vacaria, 2013. 
TABELA 5 - Parâmetros dos semivariogramas cruzados ajustados das variáveis: volume de copa (VC) versus diâmetro de caule (DC) com número diferente de amostras de volume de copa (VC) da variedade Maxi Gala, na safra 2012. Vacaria, 2013.

\begin{tabular}{|c|c|c|c|c|c|c|c|c|}
\hline Variáveis & Modelo & $\mathrm{C}$ & Co & $\mathrm{Co}+\mathrm{C}$ & $\mathrm{Co} /(\mathrm{Co}+\mathrm{C})$ & Ao & Classif. & $\mathrm{r}^{2}$ \\
\hline & & & & $\%$ & & $\mathrm{~m}$ & - & - \\
\hline \multicolumn{9}{|c|}{ Safra 2012} \\
\hline $\mathrm{VC}$ & GAUS & 2,439 & 0,001 & 2,44 & 0,0004 & 15,90 & Forte & 0,78 \\
\hline VCxDC & GAUS & 8,640 & 0,01 & 8,65 & 0,0010 & 14,20 & Forte & 0,71 \\
\hline $\operatorname{VCxDC}(-5)$ & GAUS & 8,970 & 0,01 & 8,98 & 0,0010 & 13,90 & Forte & 0,67 \\
\hline $\operatorname{VCxDC}(-10)$ & GAUS & 9,620 & 0,01 & 9,63 & 0,0010 & 13,80 & Forte & 0,65 \\
\hline VCxDC (-15) & GAUS & 9,400 & 0,01 & 9,41 & 0,0010 & 13,10 & Forte & 0,53 \\
\hline $\operatorname{VCxDC}(-20)$ & GAUS & 9,440 & 0,01 & 9,45 & 0,0010 & 12,70 & Forte & 0,49 \\
\hline VCxDC (-25) & & 0 & 0,83 & 0,83 & 1 & 91,07 & E.P. Puro & 0,19 \\
\hline
\end{tabular}

Efeito Pepita $(\mathrm{Co})$ : semivariância no ponto zero; Patamar $(\mathrm{Co}+\mathrm{C})$ : semivariância a um espaço maior que a distância do alcance; Alcance (Ao): distância após a qual os valores das propriedades não são espacialmente correlacionados; Dependência espacial (forte $<25 \%$; moderada 25 a $75 \%$; fraca $>75 \%$ a 100 e $>100$ efeito pepita puro); Modelo ajustado aos semivariogramas experimentais: Gaussiano.

\section{CONCLUSÃO}

Pela técnica da cokrigagem dos parâmetros PROD (variável) e DC (covariável), na safra de 2011, houve redução de 15 amostras do parâmetro PROD.

Pela técnica da cokrigagem dos parâmetros VC (variável) e DC (covariável), na safra de 2011, não houve dependência espacial entre eles. Na safra de 2012 houve redução de 20 amostras do parâmetro $\mathrm{VC}$.

A técnica da cokrigagem pode ser uma ferramenta da AP a ser utilizada pela cultura da macieira no levantamento de informações. Verificouse que houve resposta e redução na coleta de amostras das variáveis mais difíceis.

Maiores estudos nesta técnica, bem como buscar novas ferramentas são necessários.

\section{AGRADECIMENTOS}

Agradecemos a CAPES, empresa Rasip, grupo de fruticultura do CAV UDESC pelo apoio ao projeto.

\section{REFERÊNCIAS}

BEIGUELMAN, B. Curso prático de bioestatística. 4.ed.rev. Ribeirão Preto: Sociedade Brasileira de Genética, 1996. 274p.

BIFFI, L.J.; RAFAELI NETO, S. L. Comportamento espacial de variáveis agronômicas da maçã 'Fuji' durante dois anos de observações no planalto serrano de Santa Catarina. Revista Brasileira de Fruticultura, Jaboticabal, v.30, n.4, p.975-980, 2008.

BITTENCOURT, C. C.; MATTEI, L. F.; SANT'ANNA, P. R.; LONGO, O. C.; BARONE, F. M. A cadeia produtiva da maçã em Santa Catarina: competitividade segundo produção e packing house. Revista de Administração Pública, São Paulo, v. 45, n. 4, p. 1199-1222, 2011.

CAMBARDELA, C. A. et al. Fieldscale variability of soil properties in central Iowa soils. Soil Science Society of America Journal, Madison, v.58, n.5, p.1501-1511, 1994.

FIORAVANÇO, J. C. MAÇÃ BRASILEIRA: Da importação à auto-suficiência e exportação - A tecnologia como fator determinante. Informações Econômicas, São Paulo, v.39, n.3, p.56-67, 2009. 
LANDIM, P.M.B.; STURARO, J.R.; MONTEIRO, R. C. Krigagem ordinária para situações com tendência regionalizada. Rio Claro: DGA,IGCE,UNESP, 2002. 12 p. (Texto Didático, 6). Disponível em: $<$ http://www.rc.unesp.br/igce/ aplicada/textodi.html $>$. Acesso em: 27 jun. 2011.
SIQUEIRA, G.M.; VIEIRA, S.R.; DECHEN, S.C.F. Variabilidade espacial da densidade e da porosidade de um latossolo vermelho eutroférrico sob semeadura direta por 20 anos. Bragantia, Campinas, v.68, n.3, p.751.759, 2009.

WARRICK, W.N.; NIELSEN, D.R. Spatial variability of soil physical properties in the field. In: HILLEL, D. (Ed.). Applications of soil physics. New York: Academic Press, 1980. p.319-344. 